\title{
SYSTEMS WITH EXTENDED AVAILABILITY AND SWITCHOVER DELAYS
}

\author{
V.S.S. Yadavalli ${ }^{1}$, M.A. Shahul Hameed ${ }^{2}$, L. Prathaban $^{3}$ and K. Thaga ${ }^{4}$ \\ ${ }^{1}$ Department of Industrial and Systems Engineering \\ University of Pretoria, South Africa \\ sarma.yadavalli@up.ac.za \\ ${ }^{2}$ A.G.A. College, India \\ ${ }^{3}$ M.G.A. College, India \\ ${ }^{4}$ University of Botswana, Botswana
}

\begin{abstract}
A system with extended availability and switchover delays with a single repair facility is considered, with a random extended available period having a switching delay from online failure to the repair of the facility. Identifying suitable regeneration points, several operating characteristics, such as the moment generating function of the total uptime, mean, and mean square number of various events, are obtained, apart from reliability and availability functions.
\end{abstract}

\section{OPSOMMING}

'n Stelsel met verlengde beskikbaarheid, oorskakelvertraging met 'n enkele herstelfasiliteit en 'n toevalverlengde beskikbaarheidsperiode met oorskakeling vanaf intydse mislukking tot herstel, word behandel. Deur geskikte regenerasiepunte te identifiseer, kan verskeie bedryfseienskappe (benewens betroubaarheid en beskikbaarheid) soos 'n momentgenerende funksie vir totale beskikbare tyd, gemiddelde en gemiddelde kwadraatwaardes vir verskeie gebeurtenisse, bepaal word. 


\section{INTRODUCTION}

Repair maintenance and standby redundancy are well known methods for improving the operating characteristics of a system. In the analysis of redundant repairable systems, it is generally assumed that the system becomes unavailable immediately after breakdown. Von Ellenrider \& Levine (1966) considered a one-unit system in which the system performance is maintained for a fixed length of time even after failure. Ross \& Schechtman (1979) have generalized the above concept and studied an n-unit parallel system in which each unit has the above-mentioned property. In other words, the system after breakdown is still available for a fixed length of time. They obtained the Mean Time to System Failure (MTSF) when the life and replacement times of the individual units are exponential for the general case, and for a special case of a one-unit system, the expression for the moment generating function (MGF) for the time to system failure is given when the life and replacement times constitute two independent families of IID random variables. Bhat \& Gururajan (1993), El-Said \& El-Sherbeny (2005) studied two unit standby redundant systems with imperfect repair (that is, assumed different failure time distributions, or more precisely, distributions with decreasing expected life times, after each repair) and excessive available period, and derived the availability and reliability functions explicitly.

Examples of systems with extended available period can be found in missile technology, where the missile loses it course and becomes ineffective only if the time of jamming exceeds a prescribed short period. Also, in refrigeration and airconditioning systems, the required temperature is maintained for a random length of time, even after the failure of the unit. Hence, it is better to consider models in which the amount of time the unit continues to maintain system performance after failure is random than those where it is constant. This aspect has been incorporated into the various models analyzed in this paper. More specifically, the system continues to be available for a random unit of time even after system failure. Such systems are referred to as systems with extended or excessive available period. These systems suffer actual breakdown only if the non-functioning period exceeds this extended available period.

All the papers mentioned above deal only with cold standby systems with an extended available period and with perfect and instantaneous switching. In this paper we deal with a warm standby system in which the switching from standby to online is instantaneous and the switching from online to repair facility is non-instantaneous, along with the extended available period.

Sections 2 and 3 give some assumptions and notation, while Section 4 studies a oneunit system in detail. Apart from reliability and availability functions, several operating characteristics, such as the moment generating function (MGF) of the total uptime, mean, and mean square number of various events, are also obtained. Section 5 is devoted to the study of a multi-component series system with exponential life times and arbitrary repair times, and the final section analyzes a two-unit warm standby redundant system. 


\section{ASSUMPTIONS}

The system considered in this paper has the following assumptions.

Each system is supported by a single repair facility (RF).

Any repaired item is like a new one.

Each system has a random extended available period B, with pdf $b$ (.).

Once the system begins to operate, the effect of the extended available period is lost. From RF to online, all the switching times are instantaneous and the switch is perfect.

There is a switching delay from online failure to the RF with pdf 1 (.).

\section{NOTATION}

f (.) : :pdf of the life time of the online unit.

$\mathrm{g}($.$) \quad :pdf of the repair time of the online unit.$

$f_{s}($.$) \quad :pdf of the life time of the standby unit.$

$g_{s}($.$) \quad :pdf of the repair time of the standby unit.$

b(.) $\quad$ :pdf of the extended available period.

1(.) :pdf of the switchover time from online failure to RF.

e-event :The event denotes that the operation of a unit begins.

$e_{0}$-event :The event that both the units are good.

$e_{1}$-event :The event of the commencement of repair of a unit while the other

unit

begins to operate.

$A_{i}(t)=\operatorname{Pr}\left\{\right.$ that the system is available at $\mathrm{t} / e_{i}$ at $\left.\mathrm{t}=0.\right\},(\mathrm{i}=0,1)$

$R_{i}(t)=\operatorname{Pr}\left\{\right.$ that the system is available in $(0, \mathrm{t}) / e_{i}$ at $\left.\mathrm{t}=0.\right\},(\mathrm{i}=0,1)$

$\otimes \quad$ :Convolution symbol

$f^{*}(s) \quad$ :Laplace transform of any arbitrary function $f(t)$.

h (.) $\quad$ :The renewal function.

\section{ONE-UNIT SYSTEM}

\section{(a) Reliability and availability analysis}

The system consists of a single unit whose life times and repair times are assumed to be two independent families of random variables with pdf $f($.$) and g($.$) respectively.$ Let the e-event denote the commencement of the operation of a unit. Assuming that the unit has started functioning at $\mathrm{t}=0$, the various functions describing the system performance are obtained. The integral equation governing the reliability function $\mathrm{R}(\mathrm{t})$ is obtained using the following mutually exclusive and exhaustive classifications:

(i) The unit has not failed up to $t$.

(ii) The unit has failed before $t$, but the switching over is completed and the repair is not completed at $t$, and $t$ lies within the extended available period. 
(iii) The unit has failed before $t$, but the switching over is completed and the repair is not over at $t$, and $t$ lies within the extended available period.

(iv) The unit has failed before $t$, and the switching over and the repair is completed within the extended available period

(At that instant the regenerative e-event occurs [Finkelstein, 1999] ).

Hence we obtain

$$
\begin{aligned}
R(t)=\bar{F}(t) & +f(t) \otimes \bar{L}(t) \bar{B}(t) \\
+ & f(t) \otimes l(t) \bar{B}(t) \otimes \bar{G}(t) \bar{B}(t) \\
& +f(t) \otimes l(t) \bar{B}(t) \otimes g(t) \bar{B}(t) \otimes R(t)
\end{aligned}
$$

Let $P_{1}(t)=\bar{L}(t) \bar{B}(t)$;

$P_{2}(t)=l(t) \bar{B}(t)$;

$P_{3}(t)=\bar{G}(t) \bar{B}(t) ;$

$P_{4}(t)=g(t) \bar{B}(t)$.

Then

$$
\begin{aligned}
R(t)=\bar{F}(t) & +f(t) \otimes P_{1}(t) \\
& +f(t) \otimes P_{2}(\mathrm{t}) \otimes P_{3}(\mathrm{t}) \\
& +f(t) \otimes P_{2}(\mathrm{t}) \otimes P_{4}(\mathrm{t}) \otimes R(t)
\end{aligned}
$$

Taking Laplace transform on both sides of (2) we get

$$
R^{*}(s)=\frac{\bar{F}^{*}(s)+f^{*}(s)\left[P_{1}^{*}(s)+P_{2}^{*}(s) P_{3}^{*}(s)\right]}{1-f^{*}(s) P_{2}^{*}(s) P_{4}{ }^{*}(s)}
$$

If $\mathrm{T}$ denotes the time to system failure (whose survival function is given by $\mathrm{R}(\mathrm{t})$ ), the mean time to system failure is given by:

$\operatorname{MTSF}=\mathrm{E}(\mathrm{T})=R^{*}(0)=\frac{E(X)+P_{1}^{*}(0)+P_{2}^{*}(0) P_{3}^{*}(0)}{1-P_{2}^{*}(0) P_{4}^{*}(0)}$

where $E(X)=\int_{0}^{\infty} x f(x) d x$.

On substituting $l(t)=\delta(t)$ and $b(t)=\delta(t-a)$, we obtain

$E\left(e^{-s T}\right)=1+s R^{*}(s)$ 


$$
=\frac{2-f^{*}(s)\left[1+\int_{0}^{a} e^{-s t} g(t) d t-\int_{0}^{a} e^{-s t} \bar{G}(t) d t\right]}{1-f^{*}(s) \int_{0}^{a} e^{-s t} g(t) d t}
$$

and

$$
E(T)=\frac{E(X)}{\bar{G}(a)}+\frac{\int_{0}^{a} x g(x) d x}{\bar{G}(a)}+a
$$

which is in agreement with the result obtained by Ross \& Schechtman (1979), when the extended available period is a constant a.

Let a cycle denote the time interval between two successive e-events. To obtain the availability function $A(t)$, we use the classification that $t$ is a point either in the first cycle or in a subsequent cycle. The former case is further divided according to whether or not the online unit has failed before t. Making use of the above mutually exclusive and exhaustive events, we obtain

$$
\begin{aligned}
A(t)=\bar{F}(t) & +f(t) \otimes \bar{L}(t) \bar{B}(t) \\
& +f(t) \otimes l(t) \bar{B}(t) \otimes \bar{G}(t) \bar{B}(t) \\
& +f_{e}(t) \otimes A(t)
\end{aligned}
$$

where $f_{e}(t)$ is the pdf of the time interval between two successive e-events, and it is given by:

$$
f_{e}(t)=f(t) \otimes l(t) \otimes g(t)
$$

( since e-events occur at the instant of repair completion)

Taking Laplace transformations on both sides of (5):

$$
A^{*}(s)=\frac{\bar{F}^{*}(s)+f^{*}(s)\left[P_{1}^{*}(s)+P_{2}^{*}(s) P_{3}^{*}(s)\right.}{1-f_{e}^{*}(s)}
$$

The steady state availability $A_{s s}$ is given by:

$$
\begin{aligned}
& A_{\mathrm{ss}}=\lim _{s \rightarrow 0} \mathrm{~s} A^{*}(\mathrm{~s})=\frac{E(X)+P_{1}^{*}(0)+P_{2}^{*}(0) P_{3}^{*}(0)}{E(X)+E(Y)+E(Z)} \\
& \text { where } E(Y)=\int_{0}^{\infty} y g(y) d y \\
& \text { and } E(Z)=\int_{0}^{\infty} z b(z) d z
\end{aligned}
$$


For illustrative purposes we consider the case in which the life time, the switchover time, and the extended available period are Erlangian of order two, and the repair time has a double exponential distribution, given by:

$$
\begin{aligned}
& f(t)=\alpha^{2} t e^{-\alpha t}(\alpha>0) \\
& b(t)=a^{2} t e^{-a t} \quad(a>0) \\
& l(t)=\beta^{2} t e^{-\beta t}(\beta>0) \\
& g(t)=\mu_{1} \mu_{2} \quad\left(e^{-\mu_{1} t}-e^{-\mu_{2} t}\right) /\left(\mu_{1}-\mu_{2}\right), \quad\left(\mu_{1}, \mu_{2}>0 \text { and } \mu_{1} \neq \mu_{2}\right)
\end{aligned}
$$

\begin{tabular}{|c|c|c|}
\hline $\mathrm{a}$ & MTSF & $A_{s s}$ \\
\hline 0.1 & 3148.92 & 0.999 \\
\hline 0.2 & 1146.15 & 0.997 \\
\hline 0.3 & 704.05 & 0.996 \\
\hline 0.4 & 526.05 & 0.995 \\
\hline 0.5 & 433.42 & 0.994 \\
\hline 0.6 & 377.82 & 0.993 \\
\hline 0.7 & 341.24 & 0.992 \\
\hline 0.8 & 315.60 & 0.991 \\
\hline 0.9 & 296.80 & 0.990 \\
\hline 1.0 & 282.52 & 0.989 \\
\hline
\end{tabular}

The numerical values of MTSF and $A_{s s}$ for various values of ' $a$ ' (for fixed $\alpha, \mu_{1}$ and $\mu_{2}$ ) are given in table 1 .

Table 1

\section{(b) Cumulative uptime}

One of the principal quantities of interest is the total time the system is 'up' (or available) in any given interval. If $Z(t)$ represents the total uptime in $(0, t)$, then the moment generating function (MGF), $M(p, t)$ of $Z(t)$, is defined by:

$M(P, t)=E\left[e^{-p Z(t)}\right]$

Starting with an e-event initially, $M(P, t)$ can be obtained using the classifications that an e-event has either occurred in $(0, t)$ or not.

The latter case can be further divided into the following exclusive and exhaustive events:

(i) The online unit has not failed up to $t$. 
(ii) The online unit has failed before t, the switching over to RF is not completed, but lies within the extended available period.

(iii) The online unit has failed before $t$, but the switching over is completed and the repair is in progress at $t$, and $t$ lies within the extended available period.

When the e-event occurs, the process regenerates.

Hence we obtain:

$$
\begin{aligned}
M(p, t)= & e^{-p t} \bar{F}(t) \\
& +e^{-p t}[f(t) \otimes \bar{L}(t) \bar{B}(t)] \\
& +e^{-p t}[f(t) \otimes l(t) \bar{B}(t) \otimes \bar{G}(t) \bar{B}(t)] \\
& +e^{-p t}[f(t) \otimes l(t) \bar{B}(t) \otimes g(t) \bar{B}(t)] \otimes M(p, t) \\
& +\int_{0}^{t} f(u) d u \int_{u}^{t} l(v-u) d v\left[\int_{u}^{v} b(w-u) d w e^{-p w}\right] \int_{v}^{t} g(z-v) M(p, t-z) d z \\
& +\int_{0}^{t} f(u) d u \int_{u}^{t} l(v-u) d v \bar{B}(v-u) \int_{v}^{t} g(z-v) d z\left[\int_{v}^{z} b(w-u) e^{-p w}\right] M(p, t-z) d z
\end{aligned}
$$

Defining $M^{*}(p, s)=\int_{0}^{\infty} e^{-s t} M(p, t) d t$, we obtain:

$$
\begin{aligned}
M^{*}(p, s)=\bar{F}^{*}(p+s) & +f^{*}(p+s) P_{1}^{*}(p+s) \\
& +f^{*}(p+s) P_{2}^{*}(p+s) P_{3}^{*}(p+s) \\
& +f^{*}(p+s) P_{2}^{*}(p+s) P_{4}^{*}(p+s) M^{*}(p, s) \\
& +f^{*}(p+s) K(p, s) M^{*}(p, s) \\
& +f^{*}(p+s) P_{2}^{*}(p+s) J(p, s) M^{*}(p, s)
\end{aligned}
$$

where

$K(p, s)=\int_{0}^{\infty} e^{-s t} l(t) d t \int_{0}^{t} e^{-p u} b(u) d u \int_{0}^{u} e^{-p v} g(v) d v$ 
and $J(p, s)=\int_{0}^{\infty} e^{-s t} g(t) d t \int_{0}^{t} e^{-p u} b(u) d u$

and $P_{1}, P_{2}, P_{3}$ and $P_{4}$ are as defined in (1).

From (8) we have:

$$
M^{*}(p, s)=\frac{\bar{F}^{*}(p+s)+f^{*}(p+s)\left[P_{1}^{*}(p+s)+P_{2}^{*}(p+s) P_{3}^{*}(p+s)\right]}{1-f^{*}(p+s)\left[P_{2}^{*}(p+s) P_{4}^{*}(p+s)+K(p, s)+P_{2}^{*}(p+s) J(p, s)\right]}
$$

The various moments of the total uptime distribution can be obtained from the derivatives of the above functions.

\section{(c) Mean and mean square number of various events}

Apart from the e-event already defined, we introduce two more events as follows. Let a-event denote the instant at which the unit fails, and b-event the instant at which the system breaks down (that is, the instant at which the extended available period is over while the unit is undergoing repair). Also let $N_{a}(x, t) ; N_{b}(x, t)$; and $N_{e}(x, t)$ denote the number of $a, b$ and e events respectively in $(x, x+t)$. In this section, we obtain the expressions for the first and second order product densities of the three events respectively.

Starting with an e-event initially, define:

$h_{r}(t)=\lim _{\Delta \rightarrow 0} \operatorname{Pr}\left[N_{r}(t, \Delta)=1\right] / \Delta, \mathrm{r}=\mathrm{a}, \mathrm{b}, \mathrm{e}$ and

$h_{r s}\left(t_{1}, t_{2}\right)=\lim _{\Delta_{1}, \Delta_{2} \rightarrow 0} \operatorname{Pr}\left[N_{r}\left(t_{1}, \Delta_{1}\right)=1, N_{s}\left(t_{2}, \Delta_{2}\right)=1\right] / \Delta_{1}, \Delta_{2}, \mathrm{r}, \mathrm{s}=\mathrm{a}, \mathrm{b}$, e and $t_{1} \neq t_{2}$.

In other words, $h_{r}(t) d t$ denotes the probability that an r-event has occurred in $(\mathrm{t}$, $\mathrm{t}+\delta \mathrm{t})$, and $h_{r s}\left(t_{1}, t_{2}\right) d t_{1} d t_{2}$ denotes the probability of the occurrence of an r-event in $\left(t_{1}, t_{1}+\delta t_{1}\right)$ and an s-event in $\left(t_{2}, t_{2}+\delta t_{2}\right)$, irrespective of the events elsewhere. Hence we have (Srinivasan, 1974):

$E\left[N_{r}(0, t)\right]=\int_{0}^{t} h_{r}(u) d u$

and

$$
E\left[\left\{N_{r}(0, t)\right\}^{2}\right]=\int_{0}^{t} \int_{0}^{t} h_{r r}(u, v) d u d v+\int_{0}^{t} h_{r}(u) d u
$$

$$
=2 \int_{0}^{t} d u \int_{0}^{t} h_{r r}(u, v) d u d v+\int_{0}^{t} h_{r}(u) d u
$$


We obtain the equations satisfied by the various product densities $h_{r}(t),(\mathrm{r}=\mathrm{a}, \mathrm{b}, \mathrm{e})$ by using the classifications that the r-event which occurred in $(t, t+\delta t)$ (starting with an e-event initially) is the first one or the subsequent one. This yields:

$h_{e}(t)=f_{e}(t)+h_{e}(t) \otimes f_{e}(t)$

$h_{a}(t)=f_{a}(t)+h_{e}(t) \otimes f_{a}(t)$

$h_{b}(t)=f_{b}(t)+h_{e}(t) \otimes f_{b}(t)$

Where

$$
\begin{aligned}
& f_{e}(t)=f(t) \otimes l(t) \otimes g(t) \\
& f_{a}(t)=f(t) \otimes l(t) \\
& f_{b}(t)=f(t) \otimes(l(t) \bar{B}(t)) \otimes(b(t) \bar{G}(t))+f(t) \otimes(\bar{L}(t) b(t))
\end{aligned}
$$

Taking Laplace transform on both sides of equation (10):

$$
h_{e}^{*}(s)=\frac{f_{e}^{*}(s)}{1-f_{e}^{*}(s)}
$$

from which first order product density is obtained as:

$$
\begin{aligned}
\lim _{t \rightarrow \infty} h_{e}(t) & =\lim _{s \rightarrow 0} s h_{e}^{*}(s) \\
& =[E(X)+E(Y)+E(Z)]^{-1}
\end{aligned}
$$

Taking Laplace transform on both sides of equation (11):

$$
\begin{aligned}
h_{a}^{*}(s) & =f_{a}^{*}(s)\left[1+h_{e}^{*}(s)\right] \\
& =\frac{f_{a}^{*}(s)}{1-f_{e}^{*}(s)} \quad\{\text { using }(13)\}
\end{aligned}
$$

The stationary first order product density of a-event is obtained as:

$$
\begin{aligned}
& \lim _{t \rightarrow \infty} h_{a}(t)=\lim _{s \rightarrow 0} s h_{a}{ }^{*}(s) \\
& =\frac{s{f_{a}}^{*}(s)}{1-f_{e}{ }^{*}(s)}=[E(X)+E(Y)+E(Z)]^{-1}
\end{aligned}
$$


Let $\quad P_{5}(t)=b(t) \bar{G}(t)$

and $\quad P_{6}(t)=\bar{L}(t) b(t)$.

then $f_{b}{ }^{*}(s)=f^{*}(s) P_{2}^{*}(s) P_{5}^{*}(s)+P_{6}^{*}(s)$

Similarly, taking Laplace transform on both sides of (12), we have:

$$
\begin{aligned}
& h_{b}{ }^{*}(s)=\frac{f_{b}{ }^{*}(s)}{1-f_{e}{ }^{*}(s)} \\
& =\frac{f^{*}(s)\left\lfloor P_{2}^{*}(s) P_{5}^{*}(s)+P_{6}^{*}(s)\right\rfloor}{1-f_{e}{ }^{*}(s)}
\end{aligned}
$$

Therefore the stationary first order product density of b-event is obtained as:

$$
\begin{aligned}
& \lim _{t \rightarrow \infty} h_{b}(t)=\lim _{s \rightarrow 0} s h_{b}{ }^{*}(s) \\
& =\lim _{s \rightarrow 0}\left[\frac{s f^{*}(s)\left[P_{2}^{*}(s) P_{5}^{*}(s)+P_{6}^{*}(s)\right.}{1-f_{e}^{*}(s)}\right] \\
& =\frac{\left[P_{2}^{*}(0) P_{5}^{*}(0)+P_{6}^{*}(0)\right]}{E(X)+E(Y)+E(Z)}
\end{aligned}
$$

Now, we shall proceed to write expressions for the various second order product densities. There are nine such functions associated with the three events $e, a$ and $b$. Since the e-events are regenerative in nature, we have ( starting with an e-event initially):

$h_{e e}(t, t+\tau)=h_{e}(t) h_{e}(\tau)$

$h_{e a}(t, t+\tau)=h_{e}(t) h_{a}(\tau)$

and $h_{e b}(t, t+\tau)=h_{e}(t) h_{b}(\tau)$

In the stationary case we have:

$\lim _{t \rightarrow \infty} h_{e e}(t, t+\tau)=[E(X)+E(Y)+E(Z)]^{-1} h_{e}(\tau)$

$\lim _{t \rightarrow \infty} h_{e \mathrm{a}}(t, t+\tau)=[E(X)+E(Y)+E(Z)]^{-1} h_{a}(\tau)$

$\lim _{t \rightarrow \infty} h_{e \mathrm{~b}}(t, t+\tau)=[E(X)+E(Y)+E(Z)]^{-1} h_{b}(\tau)$ 
Similarly, since the a-events are regenerative in nature, it can be easily been seen that:

$$
\begin{aligned}
& h_{a a}(t, t+\tau)=h_{a}(t) h_{e}(\tau) \\
& h_{a e}(t, t+\tau)=h_{a}(t)\left[\mathrm{g}(\tau)+\mathrm{g}(\tau) \otimes h_{e}(\tau)\right. \\
& h_{a b}(t, t+\tau)=h_{a}(t)\left[f_{b}(\tau)+f_{b}(\tau) \otimes h_{e}(\tau)\right.
\end{aligned}
$$

From the above equations, as $\mathrm{t} \rightarrow \infty$, we obtain:

$$
\begin{aligned}
& \lim _{t \rightarrow \infty} h_{a a}(t, t+\tau)=[E(X)+E(Y)+E(Z)]^{-1} h_{e}(\tau) \\
& \lim _{t \rightarrow \infty} h_{a e}(t, t+\tau)=[E(X)+E(Y)+E(Z)]^{-1}\left[\mathrm{~g}(\tau)+\mathrm{g}(\tau) \otimes h_{e}(\tau)\right] \\
& \lim _{t \rightarrow \infty} h_{a b}(t, t+\tau)=[E(X)+E(Y)+E(Z)]^{-1}\left[f_{b}(\tau)+f_{b}(\tau) \otimes h_{e}(\tau)\right]
\end{aligned}
$$

The second order product density $h_{b b}(t, t+\tau)$ for the b-event (which is nonregenerative in nature) can be written using the classification that the b-event in $(t, t+\delta t)$ is either the first one or a subsequent one, and also the fact that an e-event always precedes a b-event. (Note that a breakdown occurs at the instant of completion of extended available period during a repair.) This gives:

$$
\begin{aligned}
& h_{b b}(t, t+\tau)=\int_{0}^{t} f(u) b(t-u) d u \int_{t}^{t+\tau} l(v-u) d v \int_{v}^{t+\tau} g(w-v) d w h_{b}(t+\tau-w) \\
& +\int_{0}^{t} h_{e}(z) d z \int_{z}^{t} f(u-z) b(t-u) d u \int_{t}^{t+\tau} l(v-u) d v \\
& \int_{v}^{t+\tau} g(w-v) d w h_{b}(t+\tau-w)
\end{aligned}
$$

The above equation can be rewritten as:

$$
\begin{aligned}
& h_{b b}(t, t+\tau)=\int_{0}^{t} f(u) b(t-u) d u \int_{0}^{\tau} l(t+v-u) d v \int_{v}^{\tau} g(t+w-v) d w h_{b}(\tau-w) \\
& +\int_{0}^{t} h_{e}(z) d z \int_{z}^{t} f(u-z) b(t-u) d u \int_{0}^{\tau} l(t+v-u) d v \\
& \quad \int_{v}^{\tau} g(t+w-v) d w h_{b}(\tau-w)
\end{aligned}
$$




$$
\begin{gathered}
h_{b b}(t, t+\tau)=\int_{0}^{t} f(t-u) b(u) d u \int_{0}^{\tau} l(u+v) d v \int_{v}^{\tau} g(w+v) d w h_{b}(\tau-w) \\
+\int_{0}^{t} h_{e}(z) d z \int_{0}^{t-z} f(t-u-z) b(u) d u \int_{0}^{\tau} l(u+v) d v \\
\int_{v}^{\tau} g(w+v) d w h_{b}(\tau-w)
\end{gathered}
$$

Let $j(t, \tau)=b(t) \int_{0}^{\tau} l(t+v) d v \int_{v}^{\tau} g(t+w) h_{b}(\tau-w) d w$

then $h_{b b}(t, t+\tau)=f(t) \otimes j(t, \tau)+h_{e}(t) \otimes f(t) \otimes j(t, \tau)=k(t, \tau)$

Now as $\mathrm{t} \rightarrow \infty$, we get:

$$
\begin{aligned}
& h_{b b}(t, t+\tau)=\lim _{t \rightarrow \infty} k(t, \tau) \\
& =\lim _{s \rightarrow 0} s k^{*}(s, \tau) \\
& =\lim _{s \rightarrow 0} s\left[f^{*}(s) j^{*}(s, \tau)+h_{e}{ }^{*}(s) f^{*}(s) j^{*}(s, \tau)\right\rfloor
\end{aligned}
$$$$
h_{b b}(t, t+\tau)=\lim _{s \rightarrow 0} s\left[\left(f^{*}(s) j^{*}(s, \tau)\right)\left\{1+h_{e}^{*}(s)\right\}\right]
$$$$
=\lim _{s \rightarrow 0} \frac{s\left[\left(f^{*}(s) j^{*}(s, \tau)\right)\right]}{\left(1-f_{e}^{*}(s)\right)}
$$$$
=[E(X)+E(Y)+E(Z)]^{-1}\left[j^{*}(0, \tau)\right]
$$$$
=[E(X)+E(Y)+E(Z)]^{-1}\left[\int_{0}^{\infty} b(u) d u \int_{0}^{\tau} l(u+v) d v\right.
$$

$\left.\int_{v}^{\tau} g(v+w) h_{b}(\tau-w) d w\right]$

Similarly, the equation for $h_{b a}(t, t+\tau)$ and $h_{b e}(t, t+\tau)$ can be written using the classification that the b-event at $(t, t+\delta t)$ is either the first one or a subsequent one. This results in using the classification that the breakdown at $(t, t+\delta t)$ is due to the first or subsequent failure; so we can write the expression for $h_{b a}(t, t+\tau)$ and $h_{b e}(t, t+\tau)$ as:

$$
\begin{aligned}
& h_{b e}(t, t+\tau)=\int_{0}^{t} f(u) b(t-u) d u \int_{t}^{t+\tau} l(v-u) d v \int_{v}^{t+\tau} g(w-v) d w h_{e}(t+\tau-w) \\
& +\int_{0}^{t} h_{e}(z) d z \int_{z}^{t} f(u-z) b(t-u) d u \int_{t}^{t+\tau} l(v-u) d v \quad \int_{v}^{t+\tau} g(w-v) d w h_{e}(t+\tau-w)
\end{aligned}
$$

and 


$$
\begin{aligned}
& h_{b a}(t, t+\tau)=\int_{0}^{t} f(u) b(t-u) d u \int_{t}^{t+\tau} l(v-u) d v \int_{v}^{t+\tau} g(w-v) d w h_{a}(t+\tau-w) \\
& +\int_{0}^{t} h_{e}(z) d z \int_{z}^{t} f(z-u) b(t-u) d u \int_{t}^{t+\tau} l(v-u) d v \\
& \int_{v}^{t+\tau} g(w-v) d w h_{a}(t+\tau-w)
\end{aligned}
$$

Arguments similar to those employed in deriving the results (23) yield the following results for the stationary cases:

$$
\begin{aligned}
& \lim _{t \rightarrow \infty} h_{b e}(t, t+\tau)=[E(X)+E(Y)+E(Z)]^{-1}\left[\int_{0}^{\infty} b(u) d u \int_{0}^{\tau} l(u+v) d v\right. \\
& \int_{v}^{\tau} g(v+w) h_{e}(\tau-v) d v \\
& \text { and } \\
& \lim _{t \rightarrow \infty} h_{b a}(t, t+\tau)=[E(X)+E(Y)+E(Z)]^{-1}\left[\int_{0}^{\infty} b(u) d u \int_{0}^{\tau} l(u+v) d v\right. \\
& \int_{v}^{\tau} g(v+w) h_{a}(\tau-v) d v
\end{aligned}
$$

\section{MODULAR SYSTEM}

In this system we consider the case where the unit itself consists of $\mathrm{n}$ dissimilar components connected in series, in which the failure rate of the $i^{\text {th }}$ component $(1 \leq \mathrm{i}$ $\leq \mathrm{n})$ is constant equal to $\alpha_{i}(>0)$ and the corresponding pdf of the repair time is an arbitrary function $g_{i}(),.(1 \leq \mathrm{i} \leq \mathrm{n})$. Moreover, it is assumed that, during the repair of any individual component, the remaining operable component do not fail. Though the system can be treated as a one-unit system with life time being the minimum of the individual component life times, its repair time can be any one of the n-type of repair times, depending on the component which causes the failure. Hence the separate treatment.

In writing the equations for the various functions, we assume that e-event (commencement of operation of the unit) has occurred at $t=0$. To obtain the equation for $\mathrm{R}(\mathrm{t})$, we use the following mutually exclusive and exhaustive classification.

(i) None of the components that commenced operation at $t=0$ has failed until $t$.

(ii) One of the components of the unit that commenced operation at $t=0$ has failed before $t$, the switching of the unit to RF is not over, and $t$ lies within the random extended available period. 
(iii) One of the components has failed before $t$, and the repair for the failed component is not over, and $t$ lies within the extended available period.

(iv) One of the components has failed before t, the switching to RF is completed, and the repair for the failed component is over before the extended available period ends (at which instant an e-event occurs).

This yields the reliability function:

$$
\begin{aligned}
& R(t)=e^{-\gamma t}+\sum_{i=1}^{n} \alpha_{i} \int_{0}^{t} e^{-\gamma u} \bar{L}(t-u) \bar{B}(t-u) d u \\
& +\sum_{i=1}^{n} \alpha_{i} \int_{0}^{t} e^{-\gamma u} d u \int_{u}^{t} l(v-u) \bar{G}_{i}(t-v) \bar{B}(t-u) d v \\
& +\sum_{i=1}^{n} \alpha_{i} \int_{0}^{t} e^{-\gamma u} d u \int_{u}^{t} l(v-u) g_{i}(w-v) \bar{B}(w-u) d w R(t-w)
\end{aligned}
$$

where $\gamma=\sum_{i=}^{n} \alpha_{i}$.

Let $P(t)=\bar{L}(t) \bar{B}(t)$

$P_{i}(t)=l(t) \overline{G_{i}}(t) \bar{B}(t)$

and $\quad Q_{i}(t)=l(t) \mathrm{g}_{\mathrm{i}}(t) \bar{B}(t)$

then (26) becomes:

$$
\begin{aligned}
& R(t)=e^{-\gamma t}+\sum_{i=1}^{n} \alpha_{i}\left[e^{-\gamma t} \otimes P(t)\right] \\
& +\sum_{i=1}^{n} \alpha_{i}\left[e^{-\gamma t} \otimes P_{i}(t)\right] \\
& +\sum_{i=1}^{n} \alpha_{i}\left[e^{-\gamma t} \otimes Q_{i}(t)\right] \otimes R(t)
\end{aligned}
$$

Taking Laplace transform on both sides of the above equation:

$$
R^{*}(s)=\frac{1}{\gamma+s}+\frac{\sum_{i=1}^{n} \alpha_{i}}{\gamma+s}\left[P^{*}(s)+P_{i}^{*}(s)+Q_{i}^{*}(s) R^{*}(s)\right]
$$

Hence $\quad R^{*}(s)=\frac{\frac{1}{\gamma+s}+\frac{\sum_{i=1}^{n} \alpha_{i}}{\gamma+s}\left[P^{*}(s)+P_{i}^{*}(s)\right]}{1-\frac{\sum_{i=1}^{n} \alpha_{i}}{\gamma+s}\left(Q_{i}{ }^{*}(s)\right)}$ 


$$
=\frac{1+\sum_{i=1}^{n} \alpha_{i}\left[P^{*}(s)+P_{i}^{*}(s)\right]}{(\gamma+s)-\sum_{i=1}^{n} \alpha_{i} Q_{i}^{*}(s)}
$$

The mean time to system failure, starting initially with an e-event, is given by:

$$
\operatorname{MTSF}=R^{*}(0)=\left[\frac{1+\sum_{i=1}^{n} \alpha_{i}\left[P^{*}(0)+P_{i}^{*}(0)\right]}{\gamma-\sum_{i=1}^{n} \alpha_{i} Q_{i}^{*}(0)}\right]
$$

Similarly, the equation for the availability function $\mathrm{A}(\mathrm{t})$ can be written using the classification that the next regenerative event has either occurred in $(0, t)$ or not. In the latter case we have:

(i) none of the online units has failed until t .

(ii) one of the components has failed before $t$ and the switching to $\mathrm{RF}$ is in progress and the extended available period goes beyond $t$.

(iii) one of the components has failed before t, the switching to RF is over, the repair of the failed unit is in progress, and the extended available period goes beyond $\mathrm{t}$.

Hence we have:

$$
\begin{aligned}
& A(t)=e^{-\gamma t}+\sum_{i=1}^{n} \alpha_{i} \int_{0}^{t} e^{-\gamma u} \bar{L}(t-u) \bar{B}(t-u) d u \\
& +\sum_{i=1}^{n} \alpha_{i} \int_{0}^{t} e^{-\gamma u} d u \int_{u}^{t} l(v-u) \bar{G}_{i}(t-v) \bar{B}(t-u) d v \\
& +\sum_{i=1}^{n} \alpha_{i} e^{-\gamma t} \otimes l(t) \otimes g_{i}(t) \otimes A(t) \\
& A(t)=e^{-\gamma t}+\sum_{i=1}^{n} \alpha_{i}\left[\left(e^{-\gamma t} \otimes P(t)\right)+\left(e^{-\gamma t} \otimes P_{i}(t)\right)\right. \\
& \left.\quad+\left(e^{-\gamma t} \otimes M_{i}(t) \otimes A(t)\right)\right]
\end{aligned}
$$

where $\quad M_{i}(t)=l(t) \otimes g_{i}(t)$

Taking Laplace transform on both sides of (31), we have: 


$$
A^{*}(s)=\frac{\frac{1}{\gamma+s}+\frac{\sum_{i=1}^{n} \alpha_{i}}{\gamma+s}\left[P^{*}(s)+P_{i}^{*}(s)\right]}{1-\frac{\sum_{i=1}^{n} \alpha_{i}}{\gamma+s}\left(M_{i}^{*}(s)\right)}
$$

The steady state availability $A_{s s}$ is given by:

$$
A_{s s}=\lim _{s \rightarrow 0} s A^{*}(s)=\frac{1+\sum_{i=1}^{n} \alpha_{i}\left[P^{*}(0)+P_{i}^{*}(0)\right]}{\left(1+\sum_{\mathrm{i}=1}^{\mathrm{n}} \alpha_{i} m_{i}\right)}
$$

where $m_{i}$ is the mean repair time of the $\mathrm{i}^{\text {th }}$ component.

The equation for MGF for the total up time $M(p, t)$ in any interval $(0, t)$ can be obtained.

Using arguments similar to that of the equation (7) yields:

$$
\begin{aligned}
& M(p, t)=e^{-(p+\gamma t)} \\
& +e^{-p t} \sum_{i=1}^{n} \alpha_{i} \int_{0}^{t} e^{-\gamma u} d u \bar{L}(t-u) \bar{B}(t-u) d u \\
& +e^{-p t} \sum_{i=1}^{n} \alpha_{i} \int_{0}^{t} e^{-\gamma u} d u \int_{u}^{t} l(v-u) \bar{G}_{i}(t-v) \bar{B}(t-v) d v \\
& +\sum_{i=1}^{n} \alpha_{i} \int_{0}^{t} e^{-\gamma u} d u \int_{u}^{t} l(v-u) d v \int_{v}^{t} g_{i}(w-v) \bar{B}(w-v) d v M(p, t-w) d w \\
& +\sum_{i=1}^{n} \alpha_{i} \int_{0}^{t} e^{-\gamma u} d u \int_{u}^{t} l(v-u) d v\left[\int_{u}^{v} b(z) d z e^{-(z-v) s}\right]_{v}^{t} g_{i}(w-v) d v M(p, t-w) d w \\
& +\sum_{i=1}^{n} \alpha_{i} \int_{0}^{t} e^{-\gamma u} d u \int_{u}^{t} l(v-u) d v \bar{B}(w-v) e^{-(v-u) s} \int_{v}^{t} g_{i}(w-v) d w \\
&
\end{aligned}
$$

After some calculations by taking Laplace transformations of (34), we obtain: 


$$
M^{*}(p, s)=\left[\frac{1+\sum_{i=1}^{n} \alpha_{i} P^{*}(s+p)+\sum_{i=1}^{n} \alpha_{i} P_{i}^{*}(s+p)}{\gamma+s+p-\sum_{i=1}^{n} \alpha_{i} Q_{i}^{*}(s+p)-\sum_{i=1}^{n} \alpha_{i} K_{i}(p, s)-\sum_{i=1}^{n} \alpha_{i} L_{i}(p, s)}\right]
$$

where

$$
\begin{aligned}
& M^{*}(p, s)=\int_{0}^{\infty} M(p, t) e^{-s t} d t \\
& K_{i}(p, s)=\int_{0}^{\infty} e^{-s t} l(t) b(t) d t \int_{0}^{t} g_{i}(u) b(u) e^{-p u} d u
\end{aligned}
$$

And

$$
L_{i}(p, s)=\int_{0}^{\infty} e^{-s t} l(t) \bar{B}(t) d t \int_{0}^{t} g_{i}(u) b(u) e^{-p u} d u
$$

The knowledge of $M^{*}(p, s)$ will enable us to obtain various moments of the cumulative uptime distributions.

\section{TWO-UNIT STANDBY REDUNDANT SYSTEM}

In this section we consider a two-unit redundant system with a single RF with switchover delay from online to RF. In addition, the system will be available for a random number of time $\mathrm{B}$ from the instant of failure of a unit, while the other unit is undergoing repair. Since the system has the extended available period property, the system is good at any time instant $t$ if at least one of the two identical units is good at $t$, or the extended available period commencing from the latest failure instant is not over until t. The system is supported by a single RF; when a unit operating online fails, it is replaced by the standby unit if it is good, or as and when it is available after repair. Let $e_{0}$ (at $\mathrm{t}$ ) denote the event that both events are good and $e_{1}$ (at $\mathrm{t}$ ) denote the event of the commencement of repair of a unit while the other unit begins to operate. Also let $f(t)$ and $f_{s}(t)$ denote the pdf of the online and standby life times respectively. In this section we assume that the standby unit has a constant failure rate $\beta$ - that is, $f_{s}(t)=\beta e^{-\beta t},(\beta>0)$. Let $g(t)$ and $g_{s}(t)$ denote the pdf of the online and standby repair times respectively.

\section{Availability and reliability analysis}

The equation governing the reliability function $R_{1}(t)$ starting with an $e_{1}$-event initially can be written using the probability argument that the next $e_{1}$-event has either occurred or not. The case where $e_{1}$-event has not occurred can be further divided into the following cases:

i. The unit has not failed up to $t$. 
ii. The unit has failed before $t$, the switching of the failed unit to RF is not over, and the random extended available period is not over until $\mathrm{t}$.

iii. The unit has failed before $t$, the switching is over before $t$, but the extended available period is not over until $t$, and also the repair commenced at $t=0$ is not over until t.

iv. The repair that has commenced after $t=0$ (for a warm standby failure) is in progress, but the online failed unit has arrived the RF, and the extended available period is not over until $t$.

v. When the online unit has failed before $t$, the switching is over, but the extended available period is not over; at that time the standby is good.

Using these classifications we get:

$$
\begin{aligned}
& R_{1}(t)=\bar{F}(t)+f(t) \otimes(l(t) \bar{B}(t)) \\
& +f(t) \otimes(l(t) \bar{B}(t)) \otimes(\bar{G}(t) \bar{B}(t)) \\
& +\int_{0}^{t} g(u) d u \int_{u}^{t} h_{s}(v-u) d u \int_{v}^{t} f_{s}(w-v) \bar{G}_{s}(t-w) d w \int_{w}^{t} f_{a}(z) d z \\
& +\left[(f(t) \otimes(l(t) \bar{B}(t)))\left(g(t) \otimes \bar{F}_{s}(t)+g(t) \otimes h_{s}(t) \otimes g_{s}(t) \otimes \bar{F}_{s}(t)\right)\right. \\
& \left.+g(t) d_{1}(t)+\int_{0}^{t} g(u) d u \int_{u}^{t} h_{s}(v-u) D_{1}(t-v) d v\right] \otimes R_{1}(t)
\end{aligned}
$$

where

$$
\begin{aligned}
& f_{a}(t)=f(t) \otimes l(t) \bar{B}(t) \\
& d_{1}(t)=f(t) \otimes L(t) \bar{B}(t) \\
& h_{s}(t)=\sum_{n=1}^{\infty}\left[f_{s}(t) \otimes g_{s}(t)\right]
\end{aligned}
$$

Now the equation (35) becomes:

$$
R_{1}(t)=\bar{F}(t)+f(t) \otimes P_{1}(t)+m_{1}(t)+q_{1}(t) \otimes R_{1}(t)
$$

where

$$
\begin{aligned}
& P_{1}(t)=\bar{L}(t) \bar{B}(t) \\
& m_{1}(t)=(f(t) \otimes(l(t) \bar{B}(t))) \otimes(\bar{G}(t) \bar{B}(t))
\end{aligned}
$$


$+\int_{0}^{t} g(u) d u \int_{u}^{t} h_{s}(v-u) d u \int_{v}^{t} f_{s}(w-v) \bar{G}_{s}(t-w) d w \int_{w}^{t} f_{a}(z) d z$

and

$$
\begin{aligned}
& q_{1}(t)=\left[(f(t) \otimes(l(t) \bar{B}(t)))\left(g(t) \otimes \bar{F}_{s}(t)+g(t) \otimes h_{s}(t) \otimes g_{s}(t) \otimes \bar{F}_{s}(t)\right)\right. \\
& \left.+g(t) d_{1}(t)+\int_{0}^{t} g(u) d u \int_{u}^{t} h_{s}(v-u) D_{1}(t-v) d v\right]
\end{aligned}
$$

Taking Laplace transform on both sides of (36), we get:

$$
\text { i.e. } \quad R_{1}^{*}(s)=\left[\frac{\bar{F}_{1}^{*}(s)+f^{*}(s) P_{1}^{*}(s)+m_{1}^{*}(s)}{\left[1-q_{1}{ }^{*}(s)\right]}\right]
$$

Hence the MTSF starting with an $e_{1}$-event at $\mathrm{t}=0$ is given by:

$$
R_{1}^{*}(0)=\left[\frac{E(X)+P_{1}^{*}(0)+m_{1}^{*}(0)}{\left[1-q_{1}^{*}(0)\right]}\right]
$$

Similarly, we can obtain the equation governing the reliability function $R_{0}(t)$, starting with an $e_{0}$-event initially by using the fact that the next $e_{1}$-event has either occurred in $(0, t)$ or not. Hence we have:

$$
\begin{aligned}
& R_{0}(t)=\bar{F}(t)+f(t) \otimes(\bar{L}(t) \bar{B}(t)) \\
& +\bar{G}(t)[f(t) \otimes l(t) \bar{B}(t)] \\
& +\int_{0}^{t} g(u) d u \int_{u}^{t} h_{s}(v-u) d v \int_{v}^{t} f_{s}(w-v) \bar{G}_{s}(t-w) D_{1}(t-w) d w \\
& +\left[(f(t) \otimes l(t) \bar{B}(t))\left(g(t) \otimes \bar{F}_{s}(t)+g(t) \otimes h_{s}(t) \otimes g_{s}(t) \otimes \bar{F}_{s}(t)\right)\right. \\
& +g(t) d_{1}(t)+\int_{0}^{t} g(u) d u \int_{u}^{t} h_{s}(v-u) d v \\
& \left.\int_{v}^{t} f_{s}(w-v) g_{s}(t-w) D_{1}(t-w) d w\right] \otimes R_{1}(t) \\
& \text { Let } m_{0}=\bar{G}(t)[f(t) \otimes l(t) \bar{B}(t)] \\
& +\int_{0}^{t} g(u) d u \int_{u}^{t} h_{s}(v-u) d v \int_{v}^{t} f_{s}(w-v) \bar{G}_{s}(t-w) D_{1}(t-w) d w
\end{aligned}
$$

and 
$q_{0}=[f(t) \otimes l(t) \bar{B}(t))\left(g(t) \otimes \bar{F}_{s}(t)+g(t) \otimes h_{s}(t) \otimes g_{s}(t) \otimes \bar{F}_{s}(t)\right)$

$+g(t) d_{1}(t)+\int_{0}^{t} g(u) d u \int_{u}^{t} h_{s}(v-u) d v$

$\left.\int_{v}^{t} f_{s}(w-v) g_{s}(t-w) D_{1}(t-w) d w\right]$

The equation (39) becomes:

$$
R_{0}(t)=\bar{F}(t)+f(t) \otimes P_{1}(t)+m_{0}(t)+q_{0}(t) \otimes R_{1}(t)
$$

Taking Laplace transform on both sides of the above equation, and using (37), we have:

$$
R_{0}^{*}(s)=\bar{F}^{*}(s)+f^{*}(s) \otimes P_{1}^{*}(s)+m_{0}^{*}(s)+q_{0}^{*}(s)\left[\frac{\bar{F}_{1}^{*}(s)+f^{*}(s) P_{1}^{*}(s)+m_{1}^{*}(s)}{1-q_{1}^{*}(s)}\right]
$$

Hence the MTSF starting with an $e_{0}$-event at $\mathrm{t}=0$ is given by:

$$
R_{0}{ }^{*}(0)=\left[\frac{\left[E(X)+P_{1}^{*}(0)\right]\left[1-q_{1}^{*}(0)+q_{0}{ }^{*}(0)\right]+m_{0}{ }^{*}(0)\left[1-q_{0}{ }^{*}(0)+q_{0}{ }^{*}(0) m_{1}{ }^{*}(0)\right]}{\left(1-q_{1}{ }^{*}(0)\right)}\right]
$$

The equation for the availability function $A_{1}(t)$, starting with an $e_{1}$-event initially, is written using the mutually exclusive and exhaustive classification that (i) the unit has not failed before $t$; (ii) it has failed before $t$ and the switching and the extended available period are not over; (iii) it has failed before t, the switching is over, the failed unit queues up for repair, and the extended available period is not over; (iv) it has failed before $t$ during the repair of the other unit and the extended available period as well as the repair of the other unit is not over until $t$, and the switching to $\mathrm{RF}$ is over before $\mathrm{t}$.

$$
A_{1}(t)=\bar{F}(t)+f(t) \otimes P_{1}(t)+m_{1}(t)+f_{w_{1}}(t) \otimes A_{1}(t)
$$

where

$$
\begin{aligned}
& m_{1}(t)=(f(t) \otimes(l(t) \bar{B}(t))) \otimes(\bar{G}(t) \bar{B}(t)) \\
& +\int_{0}^{t} g(u) d u \int_{u}^{t} h_{s}(v-u) d u \int_{v}^{t} f_{s}(w-v) \bar{G}_{s}(t-w) d w \int_{w}^{t} f_{a}(z) d z \\
& \text { and } \\
& f_{w_{1}}(t)=\left[(f(t) \otimes l(t))\left(g(t) \otimes \bar{F}_{s}(t)+g(t) \otimes h_{s}(t) \otimes g_{s}(t) \otimes \bar{F}_{s}(t)\right)\right. \\
& +g(t) \int_{0}^{t} d_{2}(u) d u
\end{aligned}
$$


$\left.+\int_{0}^{t} g(u) d u \int_{u}^{t} h_{s}(v-u) d v \int_{v}^{t} f_{s}(w-v) g_{s}(t-w) d w D_{2}(t-w)\right]$

Similarly we can write:

$A_{0}(t)=\bar{F}(t)+f(t) \otimes P_{1}(t)+P_{0}(t)+f_{w_{0}}(t) \otimes A_{1}(t)$

where

$$
\begin{aligned}
& P_{0}(t)=(f(t) \otimes l(t) \bar{B}(t)) \\
& +\int_{0}^{t} h_{s}(u) d u \int_{u}^{t} f_{s}(v-u) d v \int_{v}^{t} \bar{G}_{s}(t-w) d w D_{1}(t-w)
\end{aligned}
$$

and

$$
\begin{aligned}
& f_{w_{0}}(t)=(f(t) \otimes l(t))\left[\bar{F}_{s}(t)+h_{s}(t) \otimes g_{s}(t) \otimes \bar{F}_{s}(t)\right] \\
& +\int_{0}^{t} h_{s}(u) d u \int_{u}^{t} f_{s}(v-u) g_{s}(t-v) d v D_{2}(t-v)
\end{aligned}
$$

Taking Laplace transform on both sides of (41) and (42), we have:

$$
A_{1}^{*}(s)=\left[\frac{\bar{F}^{*}(s)+f(s) P_{1}^{*}(s)+m_{1}(s)}{1-f_{w_{1}}^{*}(s)}\right]
$$

and $A_{0}{ }^{*}(s)=\bar{F}^{*}(s)+f^{*}(s) P_{1}{ }^{*}(s)+P_{0}{ }^{*}(s)+{f_{w_{0}}}^{*}(s) A_{1}{ }^{*}(s)$

Using (43) in the above equation we get:s

$$
A_{0}^{*}(s)=\left[\frac{\begin{array}{c}
{\left[\bar{F}^{*}(s)+f^{*}(s) P_{1}^{*}(s)\right]\left[1-f_{w_{1}}{ }^{*}(s)+f_{w_{0}}{ }^{*}(s)\right]+P_{0}^{*}(s)\left[1-f_{w_{1}}{ }^{*}(s)\right]} \\
+f_{w_{0}}{ }^{*}(s) m_{1}{ }^{*}(s)
\end{array}}{\left(1-f_{w_{1}}{ }^{*}(s)\right)}\right]
$$

The steady state availability $A_{s s}$ is obtained from (43) and (44) as:

$$
\begin{aligned}
& A_{s s}=\lim _{s \rightarrow 0} A_{0}{ }^{*}(s)=\lim _{s \rightarrow 0} A_{1}^{*}(s) \\
& =\lim _{s \rightarrow 0} s\left[\frac{\bar{F}^{*}(s)+f^{*}(s) P_{1}^{*}(s)+m_{1}^{*}(s)}{1-f_{w_{1}}{ }^{*}(s)}\right]
\end{aligned}
$$




$$
=\left[\frac{E(X)+P_{1}^{*}(0)+m_{1}^{*}(0)}{m}\right]
$$

where $m$ is the mean time between successive $e_{1}$-events, given by:

$m=\int_{0}^{\infty} t f_{w_{1}}(t) d t$

Also, we can obtain the cumulative uptime for this system similar to that of the arguments and derivations in the one-unit system (Section $4[\mathrm{~b}]$ ).

In conclusion, the various operating characteristics considered in this paper can also be obtained for a two modular redundant system with extended available period for the system on failure, by combining the arguments given in the last two sections.

\section{REFERENCES}

[1] Barlow, R.E. \& Proschan, F., 1965. Mathematical theory of reliability, John Wiley, New York, NY.

[2] Cox, D.R., 1962. Renewal theory, Methuen, London.

[3] Ross, S.M. \& Schechtman, J., 1979. "On the first time a separately para maintained parallel system has been down for a fixed time", Navel Research Logistics Quarterly, Vol. 26, pp. 285-289.

[4] Von Ellenrider, A. \& Levine, A., 1966. "Probability of an excessive nonfunction interval", Operations Research, Vol. 14, pp. 835-840.

[5] Bhat, K.S. \& Gururajan, M., 1993. "A two unit cold standby system with imperfect repair and excessive availability period", Microelectronics and Reliability, Vol. 33 No. 4, pp. 509-512.

[6] El-Said, K.M. \& El-Sherbeny, M.S., 2005. "Comparing of reliability characteristics between two different systems", Applied Mathematics and Computation, available online, 15, pp. 1-17.

[7] Finkelstein, M.S., 1999. "A point process model for software reliability", Reliability Engineering and System Safety, Vol. 63, pp. 67-71. 